\title{
Caracterización clínica y por imágenes de pacientes con el ventrículo izquierdo no compacto
}

\section{Alexander Londoño-Cadavidd ${ }^{\mathrm{a}, *}$, Julián Miguel Aristizábal-Aristizábal ${ }^{\mathrm{a}}$, Pedro Abad Díaz ${ }^{\mathrm{b}}$, Jorge Delgado ${ }^{\mathrm{b}}$, Sara Restrepo-Carvajal ${ }^{\mathrm{a}}$ y Mauricio Duque-Ramírez ${ }^{\mathrm{a}}$}

\author{
a CES Cardiología y Universidad CES, Medellín, Colombia \\ ${ }^{\mathrm{b}}$ Fundación Instituto de Alta Tecnología Médica de Antioquia, IATM, Medellín, Colombia
}

Recibido el 13 de octubre de 2014; aceptado el 28 de enero de 2016

Disponible en Internet el 1 de abril de 2016

\author{
PALABRAS CLAVE \\ Ventrículo no \\ compacto; \\ Cardiomiopatía; \\ Insuficiencia cardiaca
}

\begin{abstract}
Resumen
Objetivos: El ventrículo izquierdo no compacto es cada vez más diagnosticado en el mundo con impacto significativo en la morbimortalidad. No existen registros en el medio local que describan el perfil de los pacientes afectados. El objetivo es describir las características: demográficas, clínicas e imagenológicas de los pacientes diagnosticados con el ventrículo izquierdo no compacto en un registro regional.

Métodos: Estudio descriptivo de corte transversal que incluyó pacientes con sospecha del ventrículo izquierdo no compacto por la ecocardiografía y confirmación con la resonancia magnética contrastada. Se evaluaron las historias clínicas e imágenes de los pacientes obtenidas desde el año 2006 hasta el año 2013. Se analizan las características: demográficas, clínicas e imagenológicas. La caracterización anatómica y la fracción de eyección se basaron en la resonancia magnética contrastada.

Resultados: Fueron incluidos 33 pacientes con diagnóstico del ventrículo izquierdo no compacto por la resonancia magnética contrastada. El promedio de edad fue $21,9 \pm 19,8$ años, siendo la población pediátrica la más representativa (30,3\% de 1 a 10 años y 30,3\% de 11 a 20 años). Hubo concordancia diagnóstica entre la ecocardiografía y la resonancia magnética contratada del 63,6\%; los síntomas más frecuentes fueron: la disnea, el dolor torácico y las palpitaciones $(78,8,36,4$ y $33,3 \%$, respectivamente). La insuficiencia cardiaca fue el síndrome prevalente al diagnóstico $(51,5 \%)$. La mayoría de los pacientes tenían fracción de eyección del ventrículo izquierdo $£ 40 \%(57,6 \%)$, fracción de eyección del ventrículo izquierdo normal el $21,2 \%$. Las paredes apicolaterales fueron las más comprometidas $(48,3 \%)$. Los síntomas y la presencia de embolismo tuvieron relación con la severidad de la disfunción sistólica.

Conclusiones: El ventrículo izquierdo no compacto es un diagnóstico cada vez más frecuente en el medio local y su presentación y evolución son similares a series de otros países.
\end{abstract}

\footnotetext{
* Autor para correspondencia.

Correos electrónicos: alexanderlond@yahoo.com, alexanderlondonocadavid@gmail.com (A. Londoño-Cadavid).
} 
La sospecha de la ecocardiografía guarda correlación con los hallazgos en la resonancia magnética. El diagnóstico suele hacerse en infantes y adolescentes en estadios avanzados.

(c) 2016 Sociedad Colombiana de Cardiología y Cirugía Cardiovascular. Publicado por Elsevier España, S.L.U. Este es un artículo Open Access bajo la CC BY-NC-ND licencia (http:// creativecommons.org/licencias/by-nc-nd/4.0/).

\section{KEYWORDS}

Ventricular noncompaction; Cardiomyopathy; Cardiac failure.

\section{Imaging clinical characterization of patients with left ventricular noncompaction}

\begin{abstract}
Motivation: Left ventricular noncompation is increasingly diagnosed in the world, with a significant impact on morbidity and mortality. There is no local register describing the profile of affected patients. The goal is to describe demographic, clinical and imaging characteristics of patients diagnosed with left ventricular noncompaction in a regional register.

Methods: Cross-sectional descriptive study including patients with left ventricular noncompaction suspicion due to an echocardiogram, confirmed with a magnetic resonance with contrast. Patients' medical records and images obtained between 2006 and 2013 were assessed. Demographic, clinical and imaging characteristics were analyzed. Anatomical characterization and ejection fraction were based on the magnetic resonance.

Results: 33 patients diagnosed with left ventricular noncompaction via magnetic resonance were included. The average age was $21.9 \pm 19.8$, pediatric population was the most representative (30.3\% between 1 and 10 years old and $30.3 \%$ between 11 and 20 years old). There was a 63\% diagnostic coincidence between echocardiogram and magnetic resonance; most common symptoms were dyspnea, chest pain and palpitations $(78,8,36.4,33.3 \%$ respectively). Cardiac failure was the syndrome prevalent to diagnosis (51.5\%). Most patients suffered from left ventricular noncompaction $<40 \%$ (57.6\%), normal left ventricular noncompaction $21.2 \%$. Apical lateral walls were the most compromised (48.3\%). The symptoms and presence of an embolism were more related to the severity of the systolic dysfunction.

Conclusions: Left ventricular noncompaction is an increasingly common diagnosis in the local area and its presentation and progress are similar to series from other countries. Suspicion in the echocardiogram is correlated to findings in the magnetic resonance. Diagnosis in infants and adolescents is usually reached at an advanced stage.

(c) 2016 Sociedad Colombiana de Cardiología y Cirugía Cardiovascular. Published by Elsevier España, S.L.U. This is an open access article under the CC BY-NC-ND license (http:// creativecommons.org/licenses/by-nc-nd/4.0/).
\end{abstract}

\section{Introducción}

El ventrículo izquierdo no compacto (VINC), en una alteración cardiaca caracterizada por las prominentes trabeculaciones asociadas a recesos profundos que se extienden en la cavidad ventricular hasta la superficie subendocárdica con o sin disfunción ventricular y se relaciona con un impacto variable en la morbilidad y la mortalidad ${ }^{1-4}$.

La entidad puede ser reconocida en la infancia o en la adultez y hasta ahora no es claro si el VINC es una cardiomiopatía genética distinta o un epifenómeno o variante fenotípica de otras cardiomiopatías como la cardiomiopatía dilatada.

Con el mayor acceso a los servicios de salud y la alta tecnología existe un incremento exponencial en los diagnósticos de este fenotipo de miocardiopatía y su caracterización a nivel mundial como lo demuestran el creciente número de publicaciones. Aunque el VINC ha sido por mucho tiempo considerada como una cardiomiopatía no clasificada por la OMS (Organización Mundial de la Salud) y la ESC (Sociedad Europea de Cardiología), desde el año 2006 es distinguida por la AHA (Asociación Americana del Corazón) como una cardiomiopatía primaria ${ }^{5}$. Esto ilustra la imperfección en el conocimiento y delimitación de esta enfermedad aún en la definición de los criterios diagnósticos.

Existen múltiples interrogantes sin resolver alrededor del VINC: el conocimiento de sus variables clínicas, fenotípicas, epidemiológicas y pronósticas está limitado a series y descripciones de pacientes, situación extrapolada al medio local.

Los criterios diagnósticos actuales son morfológicos. La utilidad potencial de criterios funcionales no ha sido definida ${ }^{3}$.

La ecocardiografía al ser el método de mayor disponibilidad continúa siendo el eje inicial diagnóstico y de seguimiento, a pesar de las limitaciones propias como la variabilidad interobservador, requiriendo para su confirmación diagnóstica la utilización de la resonancia nuclear magnética cardiaca (RM), por sus amplias posibilidades técnicas como: las imágenes multiplanares, la cineresonancia, las técnicas de caracterización tisular, el uso de contraste y la menor variabilidad interobservador. Es por esto que 
contemplar la aproximación multimodal en las imágenes con todos sus potenciales podría ser la recomendación para esta entidad.

\section{Metodología}

\section{Pacientes}

Se incluyeron los pacientes con sospecha diagnóstica del VINC por la ecocardiografía captados desde el año 2006 hasta el año 2013, en diferentes instituciones de salud en la ciudad de Medellín y que contaran con una resonancia magnética cardiaca con o sin contraste realizadas todas en el IATM (Instituto de Alta Tecnología Médica).

Se incluyeron todos los grupos de edad y ambos sexos, excluyendo los pacientes con otras patologías cardiacas asociadas.

Se realizó la revisión de la historia clínica correspondiente a cada paciente valorando las variables demográficas y las clínicas como: la edad, el sexo, los síntomas relacionados, la historia familiar de la falla cardiaca y la muerte súbita. Los datos no disponibles en la historia clínica fueron obtenidos por medio de entrevistas telefónicas con el paciente o sus familiares.

\section{Valoración de métodos diagnósticos por imagen}

La ecocardiografía transtorácica fue el método inicial de diagnóstico en toda la población analizada. El reporte de los estudios ecocardiográficos en donde se planteó el diagnóstico inicial fue valorado por un cardiólogo clínico y se analizaron variables como: la fracción de eyección por el método de Simpson biplano, la presencia de disfunción diastólica, las paredes y los segmentos cardiacos comprometidos, la presencia de otras anormalidades ecocardiográficas descritas, así como el cumplimiento de los criterios diagnósticos propuestos ${ }^{6}$ (tabla 1 ).

Se evaluaron los reportes de RM cardiaca considerando las mismas variables anatómicas evaluadas por la ecocardiografía y se realizó un análisis comparativo de los hallazgos entre una y otra imagen en parámetros como: las paredes y los segmentos cardiacos comprometidos, la fracción de eyección, otros hallazgos cardiacos significativos y la concordancia o discordancia en el diagnóstico del VINC entre ambas modalidades diagnósticas. Para el análisis de los resultados se tuvieron en cuenta las imágenes por RM para determinar las paredes ventriculares comprometidas y la fracción de eyección obtenida por este método. Todas las imágenes por RM fueron reevaluadas por un radiólogo experto en imágenes cardiacas verificando el diagnóstico inicial por este método basado especialmente, en los criterios de Petersen ${ }^{7}$.

El grado de compromiso de la disfunción sistólica se estadificó siguiendo los criterios de la Asociación Americana de Ecocardiografía (ASE).

\section{Análisis estadístico}

Se realizó un estudio descriptivo de corte transversal. El análisis estadístico se realizó con el programa SPSS 21 (IBM Corporation, Armonk, NY). Las variables continuas son
Tabla 1 Criterios ecocardiográficos del VINC según Jenni ${ }^{6}$ y por la RM cardiaca según Petersen ${ }^{7}$. Relación NC/C (No compacta/Compacta)

\begin{tabular}{|c|c|}
\hline $\begin{array}{l}\text { Criterios por la } \\
\text { ecocardiografía }\end{array}$ & Criterios por la RM \\
\hline $\begin{array}{l}\text { Ausencia de otra } \\
\text { anormalidad cardiaca }\end{array}$ & $\begin{array}{l}\text { Dos capas de miocardio } \\
\text { identificables }\end{array}$ \\
\hline $\begin{array}{l}\text { Presencia de } 2 \text { capas una } \\
\text { no compacta y una } \\
\text { compacta delgada con } \\
\text { espacios } \\
\text { intertrabeculares } \\
\text { profundos }\end{array}$ & $\begin{array}{l}\text { Segmentos compactados y } \\
\text { no compactados medidos } \\
\text { desde cine en eje largo, en } \\
\text { el sitio con más } \\
\text { prominentes } \\
\text { trabeculaciones }\end{array}$ \\
\hline $\begin{array}{l}\text { No compactación de } \\
\text { predominio medio } \\
\text { lateral, medio inferior } \\
\text { y segmentos apicales }\end{array}$ & $\begin{array}{l}\text { Medidas deben ser } \\
\text { perpendiculares al } \\
\text { miocardio compactado }\end{array}$ \\
\hline \multicolumn{2}{|c|}{$\begin{array}{l}\text { Evidencia por doppler color de recesos intertrabeculares } \\
\text { profundos }\end{array}$} \\
\hline Relación en sístole & Relación en diástole \\
\hline $\mathrm{NC} / \mathrm{C}>2$ & $\mathrm{NC} / \mathrm{C}>2,3$ \\
\hline
\end{tabular}

expresadas como medias y desviación estándar y las variables cualitativas como frecuencias absolutas y porcentajes. Se evaluó la normalidad en las variables continuas. Se realizó una comparación de los promedios para las muestras relacionadas con la prueba de t de student y con la prueba chi cuadrado para las variables categóricas. Se consideró como estadísticamente significativo un valor de $p<0,05$.

\section{Resultados}

Se incluyeron para el estudio 46 casos de los cuales 27 ingresaron con sospecha diagnóstica del VINC por la ecocardiografía, de los cuales 20 fueron concordantes con este diagnóstico por la RM (74\%).

Se incluyeron para el análisis final 33 pacientes con criterios confirmatorios por la RM. La edad de los pacientes analizados comprendió desde 2 meses a 72 años con un promedio de $21,9 \pm 19,8$ años de los cuales $63,6 \%$ fueron hombres y $36,4 \%$ mujeres (fig. 1 ).

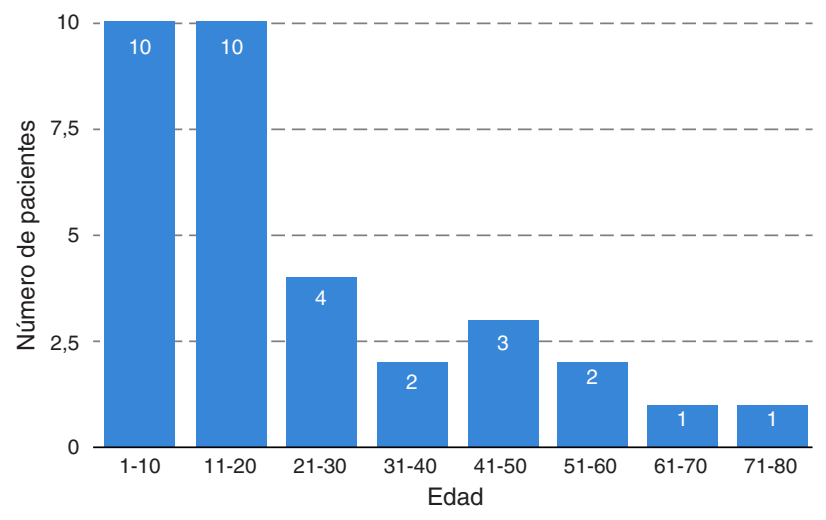

Figura 1 Distribución por edad al momento del diagnóstico. 
Tabla 2 Características clínicas de los pacientes al momento del diagnóstico

\begin{tabular}{ll}
\hline Número de pacientes con diagnóstico del VINC & 33 \\
Edad promedio y desviación estándar (años) & $21,9(19,8)$ \\
Mujeres \% & 36,4 \\
Hombres \% & 63,6 \\
Ocurrencia familiar de insuficiencia cardiaca \% & 22 \\
Sintomáticos \% & 87 \\
$\quad$ Disnea & 78,8 \\
Palpitaciones & 36,4 \\
Dolor torácico & 33,3 \\
Asintomáticos \% & 12,9 \\
Síndromes clínicos \% & \\
Insuficiencia cardiaca & 51,5 \\
Síncope & 15,1 \\
Muerte súbita & 6 \\
FEVI media y desviación estándar (\%) & $37,3(16,7)$ \\
$\quad \leq 40 \%$ & 57,6 \\
$41-50 \%$ & 21,2 \\
$\geq 51 \%$ & 21,2 \\
\hline
\end{tabular}

La mayoría de los pacientes tenía $\leq 20$ años de edad. Se analizó la presentación de los síntomas según la edad entre los mayores y los menores de 20 años. No se encontró diferencia significativa para la presentación de disnea $(p=0,839)$, dolor $(p=0,014)$, palpitaciones $(p=0,045)$ o síntomas de falla $(p=0,107)$ entre ambos grupos.

La historia familiar de la insuficiencia cardiaca estuvo presente en $22 \%$ de 9 pacientes de los que se pudo obtener información familiar, no obstante, no se identificó el diagnóstico específico del tipo de cardiomiopatía en ninguno de los pacientes analizados.

Las características epidemiológicas y clínicas de estos pacientes al momento del diagnóstico se describen en la tabla 2.

\section{Hallazgos clínicos}

Los síntomas más frecuentes referidos por los pacientes como forma de presentación inicial y que llevaron a los estudios diagnósticos fueron: la disnea en $78,8 \%$, el dolor torácico $33,3 \%$, las palpitaciones $36,4 \%$ y la fatiga $21,2 \%$. Solo 4 pacientes $(12,1 \%)$ estaban asintomáticos al momento del diagnóstico (fig. 2).

Dentro de los síndromes clínicos iniciales la insuficiencia cardiaca fue la manifestación en $51,5 \%$, el síncope en 15,1\%, la muerte súbita $6 \%$ y un episodio embólico descrito como tromboembolismo pulmonar $3 \%$.

Los síntomas y síndromes clínicos aparentemente estaban relacionados con la severidad de la insuficiencia cardiaca según la fracción de eyección medida por RM y graduada en severidad por criterios ASE (American Society of Echocardiograpy), como se demuestra en la figura 3. No obstante, para el análisis se evaluaron los síntomas y la presentación clínica según la $\mathrm{FEVI}$ en dos categorías, $\mathrm{FEVI} \leq 40 \%$ y $\mathrm{FEVI} \geq 41 \%$. No se encontró diferencia estadísticamente significativa entre los grupos para disnea $(p=0,341)$, dolor $(p=0,949)$

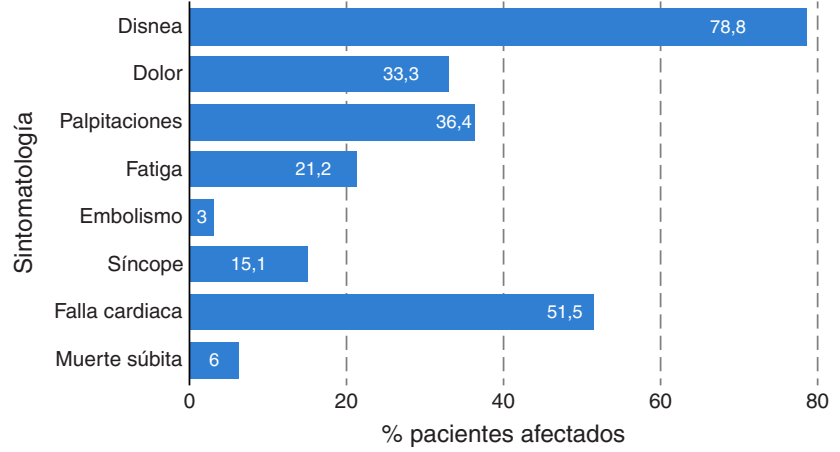

Figura 2 Frecuencia de presentación de síntomas y síndromes.

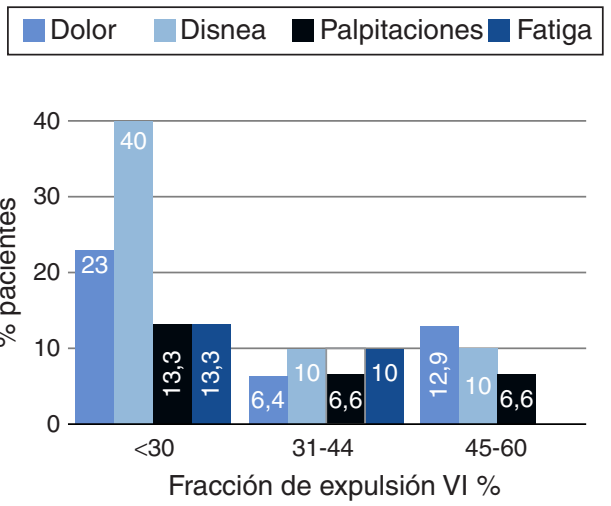

Figura 3 Relación de síntomas con la FEVI.

o palpitaciones $(p=0,811)$. Hubo diferencia significativa para la presentación de síntomas de la falla cardiaca en pacientes con $\mathrm{FEVI} \leq 40 \%$ comparado con $\mathrm{FEVI}$ normal $(p=0,002)$.

Los fenómenos embólicos reportados fueron reportados en pacientes con severa disfunción sistólica. No se documentó relación del síncope o los episodios de muerte súbita con la FEVI al momento del diagnóstico.

De todos los pacientes analizados que cumplían con los criterios de VINC por RM, solo $4(12,9 \%)$ fueron asintomáticos, coincidiendo este grupo con FEVI preservada.

El seguimiento se pudo realizar en 13 pacientes. Al momento de la entrevista telefónica con las familias o los pacientes se hallaron como complicaciones principales la muerte en 4 de 13 pacientes $(30,7 \%), 2$ recibieron trasplante cardiaco, un paciente requirió la implantación de un desfibrilador automático y otro un cardiodesfibrilador con resincronizador. Es de anotar que todos los pacientes presentaban insuficiencia cardiaca con disfunción sistólica severa.

\section{Análisis de imágenes}

Los 33 pacientes incluidos en el estudio contaron con: la ecocardiografía transtorácica, los análisis de imágenes en $2 \mathrm{D}$ y el doppler, además, con las imágenes de resonancia magnética, con equipo Philips de 1,5 Tesla y las bobinas cardiacas de 32 canales. En las imágenes por la RM estuvieron disponibles para el análisis las secuencias de cine, las imágenes 


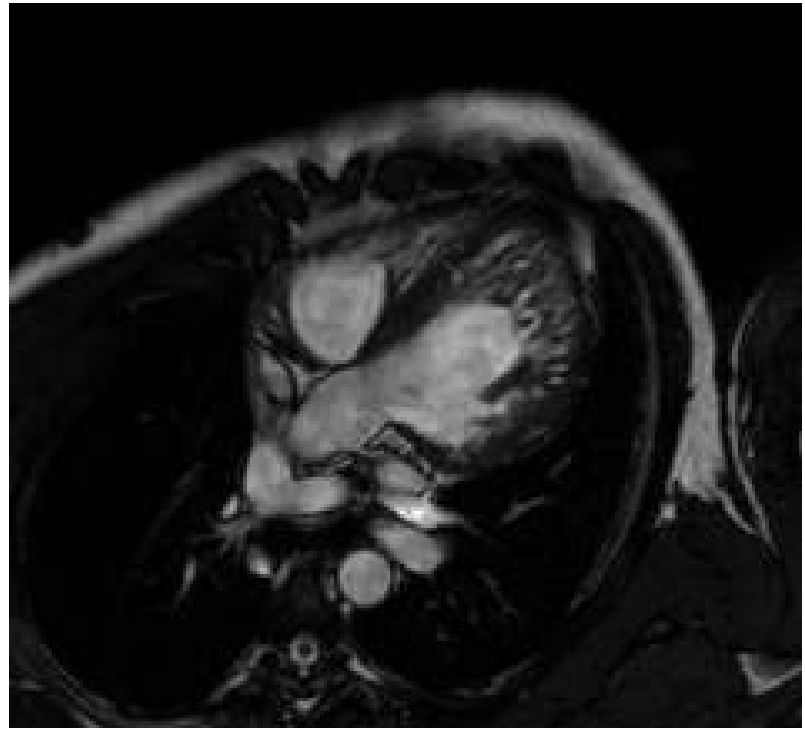

Figura 4 Imagen de RM en 4 cámaras en diástole, observando prominentes trabeculaciones apicales y en la pared lateral, con recesos profundos. No se observan trombos.

de caracterización tisular, las angiografías contrastadas y las secuencias de realce tardío.

De los 27 pacientes remitidos con sospecha diagnóstica del VINC por la ecocardiografía solo 20 cumplieron con criterios de Petersen en la RM para una coincidencia diagnóstica entre las dos modalidades de imagen del 74\%. Entre los pacientes sin concordancia diagnóstica $5(71,4 \%)$, cumplieron los criterios diagnósticos de la cardiomiopatía dilatada idiopática y $2(28,5 \%)$ fueron diagnosticados como hipertrabeculación sin criterios para el VINC.

En los hallazgos por la resonancia magnética de los 33 pacientes analizados, la pared apical y lateral fue las más frecuentemente comprometida con 16 pacientes $(48,4 \%)$ seguida por compromiso de las paredes apical, lateral, apicolateral más septal con 3 pacientes cada uno (9\%). La pared inferoposterior solo se halló comprometida en 1 paciente (3\%). De las paredes comprometidas solo 3 implicaron compromiso de los segmentos basales, los demás, comprometieron en todos los casos los segmentos medio y apical del vı (fig. 4).

Dentro de los hallazgos por la resonancia magnética (fig. 5) cabe resaltar la presencia de las trabeculaciones atravesando la cavidad ventricular de una pared a otra o pseudotendones en $9(27,2 \%)$ pacientes, el arco aórtico bovino como variante anatómica en 4 pacientes $(12,1 \%$ ) y la válvula aórtica bivalva en $3(9 \%)$ pacientes.

La función sistólica medida por RMN se encontró comprometida en la mayoría de los pacientes con un promedio de $37,3 \pm 16,7 \% ; 57,6 \%$ tenían $\mathrm{FEVI} \leq 40 \%$ y solo $21,2 \%$ tenían $\mathrm{FEVI}>50 \%$ (fig. 6).

El realce tardío solo fue hallado en $4(12,1 \%)$ de los pacientes, todos ellos con compromiso subendocárdico de los segmentos apicales comprometidos por no compactación.

Por análisis de las imágenes ecocardiográficas la disfunción diastólica fue descrita en 16 pacientes $(48,4 \%)$ siendo los trastornos de distensibilidad y relajación los más descritos (disfunción diastólica tipo ı/ıII, Clasificación ASE, American Society of Cardiology).

\section{Discusión}

Dentro de nuestro conocimiento, el presente es el registro de los pacientes con el VINC más amplio reportado en el país, contando para todos sus participantes con el análisis complementario de la ecocardiografía transtorácica y la RM cardiaca, representando una significativa muestra de la población hispana ya que los datos actuales en su gran mayoría provienen de la población caucásica.

El diagnóstico del VINC suele hacerse de forma tardía asociado con insuficiencia cardiaca $(51,6 \%)$, con sus consecuentes implicaciones pronósticas considerando que la mortalidad ha sido relacionada más con el nivel de disfunción sistólica ventricular que con el hallazgo de no compactación ${ }^{8,9}$. Esto podría asociarse en parte a los fenómenos socioeconómicos, el difícil acceso a los servicios de salud de mayor complejidad y al frecuente desconocimiento de la enfermedad y su historia natural. A pesar de ello, se encontró poca divergencia con respecto a los diferentes reportes mundiales (incidencia 30-53\%) ${ }^{2,4,10}$. Cabe resaltar que no se halló ningún caso de compromiso ventricular derecho por no compactación en cuyo caso el actual conocimiento se limita a esporádicos reportes de casos.

Otro de los fenómenos relacionado con las dificultades diagnósticas y de seguimiento en pacientes con el VINC son la poca estandarización en las guías y los consensos de los criterios diagnósticos y los elementos necesarios del reporte para unificar la información. En este caso aunque los criterios de Jenni $^{6}$ son los más aceptados en la ecocardiografía para el diagnóstico, hubo pobre descripción de estos (u otros), lo que podría explicar en parte las diferencias de correlación diagnóstica entre una y otra técnica de imágenes.

La literatura ha reportado el VINC como la tercer forma de cardiomiopatía más prevalente en los niños, prevalencia estimada entre 9,2 y $9,5 \%^{11,12}$. Esta proporción ha sido estimada en el $3 \%$ de los pacientes adultos ${ }^{2}$.

En los pacientes evaluados la edad promedio del diagnóstico fue de 21,9 años, influido de forma significativa por la inclusión de la población pediátrica en el análisis ${ }^{11-13}$. Al evaluar los datos por intervalos de edad la mayoría de pacientes son diagnosticados entre los 11 y 20 años $(35,4 \%)$, seguidos del intervalo de 1 a 10 años $(22,5 \%)$, contrario a lo

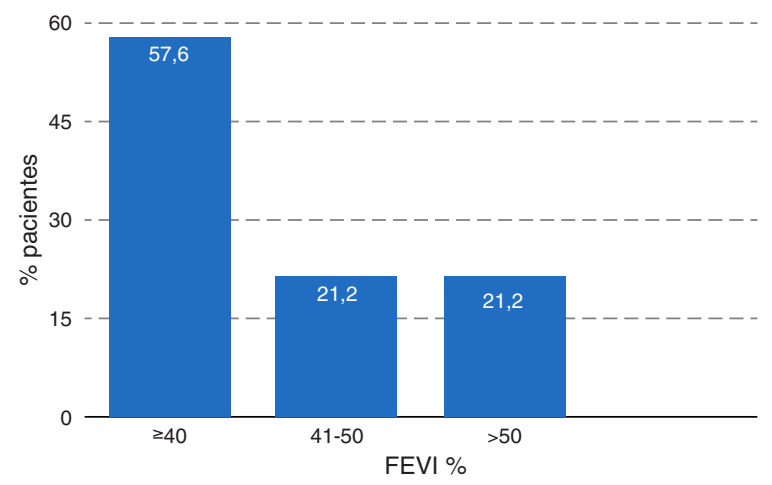

Figura 5 FEVI evaluada por resonancia magnética cardiaca contrastada. 


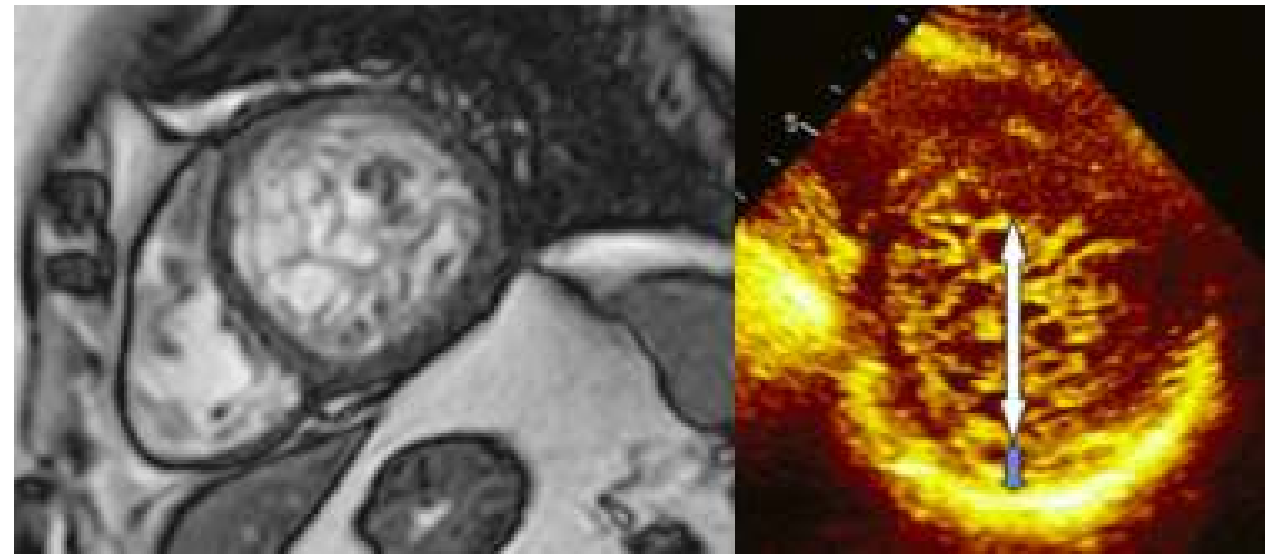

Figura 6 Imágenes por RM cardiaca y ecocardiografía en eje corto del segmento medio ventricular izquierdo que demuestra la presencia del VINC.

reportado en diferentes publicaciones en donde el segundo intervalo incluye la mayoría poblacional con el diagnóstico de esta condición clínica. Aunque en la literatura mundial existe gran divergencia entre los diferentes estudios con respecto a este tópico podríamos concluir que no varía de forma llamativa con la mayoría de reportes considerando que se incluyeron pacientes pediátricos y adultos ${ }^{13-15}$. La distribución por sexo no varía de forma significativa con los reportes mundiales con una mayor prevalencia del VINC en la población masculina $(64,5 \%$ vs. $35,4 \%)$, independientemente, de los grupos de edad analizados ${ }^{16-18}$.

De manera similar con lo publicado, la mayoría de síntomas tuvieron relación con la presencia de la disfunción sistólica ventricular izquierda y la insuficiencia cardiaca. Con respecto al tipo de síntomas, la disnea, el dolor torácico y las palpitaciones concuerdan con los síntomas predominantes descritos en diferentes estudios ${ }^{8,10}$, con un retraso en el diagnóstico hasta de 3,5 años, situación que posiblemente influye también en el pronóstico ${ }^{8}$.

Diferentes fenómenos embólicos han sido descritos en pacientes con el VINC ${ }^{19}$, en este caso fueron reportados solo en 2 casos ambos en presencia de severa disfunción sistólica. La incidencia de FA al momento del diagnóstico ha sido reportada entre el $6 \%$ y el $26 \% 6,10$ mayor a la población general, no obstante, ante la ausencia de los datos históricos y electrocardiográficos de arritmias específicas que nos permita encontrar otra relación causal, continúa como interrogante si la disfunción sistólica severa en presencia de grandes trabeculaciones y los recesos representarían una indicación para la anticoagulación como ha sido propuesto por algunos grupos de expertos. Según datos recientes la severidad de la disfunción sistólica, la presencia de fibrilación atrial (FA) y el antecedente de un evento tromboembólico previo permanecen como los principales factores de riesgo, más que la severidad de la no compactación por sí misma ${ }^{20}$. Hasta ahora continúa como una conducta aceptable la anticoagulación en presencia de: historia previa de eventos embólicos, disfunción ventricular izquierda (fracción de acortamiento < $25 \%$ y $\mathrm{FEVI}<40 \%$ ), presencia de FA o presencia de trombo ventricular ${ }^{1,16,19}$.

Los pacientes con el VINC tienen un sustrato arritmogénico. Es frecuente la presencia de arritmias supraventriculares y ventriculares ${ }^{21,22}$. La incidencia de TV sostenida ha sido estimada del $0-9 \%$ en todos los registros, mientras que la incidencia de TV no sostenida varía del 20 al 33\% en seguimientos a 2,3 a 3,8 años. La alta incidencia de TV no sostenida en estos pacientes está en relación con la presencia de FEVI reducida, sin embargo, sigue siendo baja cuando se compara con otras formas como la cardiomiopatía dilatada (60\%). A pesar de ello la TV no sostenida en este grupo de pacientes no parece influir en el riesgo de muerte súbita ${ }^{4}$. En la muestra analizada los episodios de síncope y muerte súbita no se relacionaron con la severidad de la disfunción ventricular izquierda, planteándose así la posibilidad de otros elementos funcionales y estructurales más allá de la FEVI que puedan relacionarse con las arritmias ventriculares como ha sido descrito con la presencia de cicatrices por la RM como predictores de riesgo de arritmias severas en otras cardiomiopatías, pero cuyo rol en el VINC permanece por definir. Steffel, y Cols., reportaron que la inducibilidad de TV monomórfica sostenida en el estudio electrofisiológico era baja, cuestionando la potencial utilidad del mismo en la estratificación del riesgo de muerte súbita en esta población ${ }^{21}$. Recientemente registros italianos mostraron cifras contrastantes con baja incidencia de las taquicardias supraventriculares y las ventriculares en los pacientes con el VINC ${ }^{23}$.

El uso del cardiodesfibrilador implantable también ha sido propuesto como alternativa de manejo considerando el riesgo de muerte súbita en este grupo de pacientes ${ }^{4,24}$, no obstante, para la prevención primaria y secundaria se acogen las guías actuales de manera similar a otras cardiomiopatías.

La ecocardiografía continúa siendo la modalidad diagnóstica más accesible y costo efectiva, siendo los criterios de Jenni los más aceptados; no obstante, basados en la evidencia disponible la resonancia magnética cardiaca sigue siendo la mejor modalidad de imagen considerando su capacidad multiplanar, la capacidad de usar técnicas diferentes de caracterización tisular y el uso de contraste entre otros ${ }^{3,25}$. En el estudio todos los pacientes incluidos contaron con ambas modalidades de imágenes y se encontró la concordancia diagnóstica entre la ecocardiografía y la resonancia magnética nuclear cardiaca en el $74 \%$ de los casos, hallazgo posiblemente relacionado con la poca estandarización en los parámetros ecocardiográficos usados, la no utilización 
de algunas tecnologías sugeridas en la literatura como: el uso de medio de contraste, las imágenes en $3 D^{26}$ y la mayor dependencia de esta técnica de las habilidades, la experiencia y el conocimiento del operador. Basados en la evidencia disponible, la aproximación diagnóstica sugerida actualmente es multimodal ${ }^{27}$.

Dentro de los diagnósticos diferenciales la presencia de la cardiomiopatía dilatada idiopática y el fenómeno de la hipertrabeculación sin criterios de no compactación fueron los más importantes, lo que resalta aún más la necesidad de estandarizar los criterios diagnósticos y la utilización de multimodalidad en las imágenes antes de establecer el diagnóstico.

El análisis de imágenes por la RM de las paredes ventriculares más comprometidas por la no compactación no difiere en sus hallazgos de los reportados en la literatura mundial, representando la pared apical más lateral en sus segmentos medio y apical el $45 \%$ de todos los casos, con rara ocurrencia hacia los segmentos basales. Aunque se han planteado otros hallazgos por la resonancia magnética como parte del espectro diagnóstico como: los defectos de perfusión y el realce tardío sobre el área no compactada, en este caso las imágenes por el realce tardío se evidenciaron solo en $12,9 \%$ de los pacientes a diferencia de lo descrito en la literatura con reportes hasta del $55 \%$ y no fue detectado ningún defecto en las fases de perfusión ${ }^{28,29}$. La diferencia podría relacionarse, al menos como hipótesis, con la alta proporción de los pacientes pediátricos incluidos en el estudio a diferencia de la población adulta analizada en la referencia citada. La presencia de los pseudotendones (trabeculaciones extensas de pared a pared), hallada como una de las variables anatómicas más importantes en aquel estudio (28.5\%), característica relacionada en estudios previos que puede aportar a la sensibilidad y la especificidad diagnóstica ${ }^{30,31}$.

Aún queda por aclarar la relación con otras variables anatómicas descritas como el arco aórtico bovino y la aorta bivalva $(12,9 \%$ y $9,6 \%$, respectivamente), observadas en mayor proporción a la descrita en la población general.

Existieron importantes limitaciones para el seguimiento de los pacientes por medio telefónico lo que contribuyó a la escasez de datos que permitieran evaluar el pronóstico, ello podría ser explicado tanto por factores sociales propios en nuestra población como la migración urbana y rural, así como a la ausencia de bases de datos unificadas que permitan el adecuado seguimiento de los pacientes. Estas mismas consideraciones limitaron conocer la etiología de la insuficiencia cardiaca dentro los antecedentes familiares. A pesar de lo anterior, cabe resaltar elementos importantes: los datos encontrados apoyan la ya reportada relación de la mortalidad con la severidad de la disfunción ventricular izquierda, el hallazgo no divergente en esta entidad con otro tipo de cardiomiopatías ${ }^{9,32}$, y que algunas de las terapias avanzadas para el manejo de la falla cardiaca y la prevención de la muerte súbita como el trasplante cardiaco, los resincronizadores y los desfibriladores automáticos implantables, hacen parte de las estrategias terapéuticas para los pacientes con el VINC y las indicaciones hasta ahora ajustadas a las guías $^{24,33}$.

Vale la pena resaltar la ausencia de la tamización familiar, lo que vislumbra posiblemente un profundo desconocimiento de la fisiopatología e historia natural de la enfermedad y su impacto hereditario aún en investigación. Esta entidad es un desorden genéticamente heterogéneo con formas esporádicas y familiares. Parece estar asociada con las mutaciones en las proteínas sarcoméricas, mitocondriales, citoesqueléticas y de la línea $Z^{3}$. Las formas autosómicas dominantes son más comúnmente asociadas que las formas recesivas y asociadas al cromosoma $x$. Las recurrencias familiares varían del 18 al 50\%, pero con limitaciones importantes en los diferentes estudios retrospectivos ${ }^{12,34}$. La alta tasa de trasmisión enfatiza la necesidad de adecuadas historias clínicas y familiares, así como la tamización de todos los familiares en primer grado con la ecocardiografía transtorácica como el método ideal para tal fin ${ }^{16,34}$.

\section{Limitaciones}

El estudio presenta las limitaciones propias de su diseño descriptivo de corte transversal, no obstante, por tratarse de una entidad con una muy baja prevalencia poblacional tiene relevancia en el conocimiento inicial de nuestra población. El advenimiento y refinamiento de nuevas técnicas en la ecocardiografía y la resonancia magnética incluyen parámetros más allá de los anatómicos (usados en este caso), para el diagnóstico del VINC, sin embargo, no disponemos del consenso único para los criterios diagnósticos y dentro de los disponibles, los criterios de Petersen continúan hasta el momento siendo de gran utilidad.

Los criterios de Jenni son los más aceptados en la ecocardiografía como acercamiento diagnóstico, no obstante, hubo pobre descripción de estos (u otros) en los reportes ecocardiográficos, lo que explica en parte las diferencias de correlación diagnóstica entre una y otra técnica de las imágenes.

El objetivo primario del estudio no es la determinación de los factores pronósticos y los datos específicos de seguimiento obtenidos limitan obtener otras conclusiones al respecto.

Severas limitaciones imponen a la investigación la dispersión de historias clínicas y el inadecuado seguimiento de los pacientes y sus familias por las diferentes entidades de salud.

\section{Conclusiones}

La mayoría de los datos obtenidos no difieren de los publicados en el mundo. La caracterización específica de la población colombiana puede aportar al conocimiento de la enfermedad en el medio y desarrollar comparaciones con series de otros países.

El VINC a pesar de ser una rara forma de cardiomiopatía o fenotipo de cardiomiopatía es asociado con una alta incidencia de complicaciones y un pobre pronóstico. Aunque la ecocardiografía continúa siendo la herramienta diagnóstica inicial y la resonancia magnética cardiaca el método preferido para su confirmación, es necesario refinar los criterios por estas técnicas con la posible combinación de los parámetros anatómicos y los funcionales dentro de criterios únicos y universales. El diagnóstico del VINC en nuestra población suele hacerse en infantes y adolescentes de forma tardía y en los estadios avanzados de disfunción ventricular, lo que demuestra la necesidad de una adecuada tamización familiar y seguimiento para conocer la historia natural de la 
enfermedad y probablemente impactar de forma temprana en su manejo.

\section{Responsabilidades éticas}

Protección de personas y animales. Los autores declaran que para esta investigación no se han realizado experimentos en seres humanos ni en animales.

Confidencialidad de los datos. Los autores declaran que en este artículo no aparecen datos de pacientes.

Derecho a la privacidad y consentimiento informado. Los autores declaran que en este artículo no aparecen datos de pacientes.

\section{Conflicto de intereses}

Los autores manifiestan no tener ningún conflicto de intereses.

\section{Bibliografía}

1. Almeida AG, Pinto FJ. Non-compaction cardiomyopathy. Heart. 2013;99(20):1535-42.

2. Oechslin E, Jenni R. Left ventricular non-compaction revisited: a distinct phenotype with genetic heterogeneity? European heart journal. 2011;32(12):1446-56.

3. Towbin JA, Lorts A, Jefferies JL. Left ventricular noncompaction cardiomyopathy. Lancet. 2015;386(9995):813-25.

4. Thavendiranathan P, Dahiya A, Phelan D, Desai MY, Tang WH. Isolated left ventricular non-compaction controversies in diagnostic criteria, adverse outcomes and management. Heart. 2013;99(10):681-9.

5. Maron BJ, Towbin JA, Thiene G, Antzelevitch C, Corrado D, Arnett $\mathrm{D}$, et al. Contemporary definitions and classification of the cardiomyopathies: an American Heart Association Scientific Statement from the Council on Clinical Cardiology, Heart Failure and Transplantation Committee; Quality of Care and Outcomes Research and Functional Genomics and Translational Biology Interdisciplinary Working Groups; and Council on Epidemiology and Prevention. Circulation. 2006;113(14):1807-16.

6. Jenni R, Oechslin E, Schneider J, Attenhofer Jost C, Kaufmann PA. Echocardiographic and pathoanatomical characteristics of isolated left ventricular non-compaction: a step towards classification as a distinct cardiomyopathy. Heart. 2001;86(6):666-71.

7. Petersen SE, Selvanayagam JB, Wiesmann F, Robson MD, Francis $\mathrm{JM}$, Anderson $\mathrm{RH}$, et al. Left ventricular non-compaction: insights from cardiovascular magnetic resonance imaging. Journal of the American College of Cardiology. 2005;46(1):101-5.

8. Hussein A, Karimianpour A, Collier P, Krasuski RA. Isolated Noncompaction of the Left Ventricle in Adults. Journal of the American College of Cardiology. 2015;66(5):578-85.

9. Stanton C, Bruce C, Connolly H, Brady P, Syed I, Hodge D, et al. Isolated left ventricular noncompaction syndrome. The American journal of cardiology. 2009;104(8):1135-8.

10. Habib G, Charron P, Eicher JC, Giorgi R, Donal E, Laperche T, et al. Isolated left ventricular non-compaction in adults: clinical and echocardiographic features in 105 patients. Results from a French registry. European journal of heart failure. 2011;13(2):177-85.

11. Ergul $Y$, Nisli K, Demirel A, Varkal MA, Oner N, Dursun M, et al. Left ventricular non-compaction in children and adolescents: clinical features, treatment and follow-up. Cardiology journal. 2011;18(2):176-84.

12. Lilje C, Razek V, Joyce JJ, Rau T, Finckh BF, Weiss F, et al. Complications of non-compaction of the left ventricular myocardium in a paediatric population: a prospective study. European heart journal. 2006;27(15):1855-60.

13. Chin TK, Perloff JK, Williams RG, Jue K, Mohrmann R. Isolated noncompaction of left ventricular myocardium. A study of eight cases. Circulation. 1990;82(2):507-13.

14. McMahon CJ, Pignatelli RH, Nagueh SF, Lee VV, Vaughn W, Valdes SO, et al. Left ventricular non-compaction cardiomyopathy in children: characterisation of clinical status using tissue Dopplerderived indices of left ventricular diastolic relaxation. Heart. 2007;93(6):676-81.

15. Ichida F, Hamamichi Y, Miyawaki T, Ono Y, Kamiya T, Akagi T, et al. Clinical features of isolated noncompaction of the ventricular myocardium: long-term clinical course, hemodynamic properties, and genetic background. Journal of the American College of Cardiology. 1999;34(1):233-40.

16. Lofiego C, Biagini E, Pasquale F, Ferlito M, Rocchi G, Perugini $E$, et al. Wide spectrum of presentation and variable outcomes of isolated left ventricular non-compaction. Heart. 2007;93(1):65-71.

17. Aras D, Tufekcioglu O, Topaloglu S, Ozeke O, Ergun K, Yildiz A, et al. Non-compaction cardiomyopathy associated with myocardial bridging: A frequently overlooked or misdiagnosed cardiomyopathy. European journal of heart failure. 2006;8(7):760-3.

18. Wald R, Veldtman G, Golding F, Kirsh J, McCrindle B, Benson L. Determinants of outcome in isolated ventricular noncompaction in childhood. The American journal of cardiology. 2004;94(12):1581-4.

19. Carrilho-Ferreira P, Almeida AG, Pinto FJ. Non-compaction cardiomyopathy: prevalence, prognosis, pathoetiology, genetics, and risk of cardioembolism. Curr Heart Fail Rep. 2014;11(4):393-403.

20. Stollberger C, Finsterer J. Cardiologic and neurologic findings in left ventricular hypertrabeculation/non-compaction related to wall thickness, size and systolic function. European journal of heart failure. 2005;7(1):95-7.

21. Steffel J, Kobza R, Namdar M, Wolber T, Brunckhorst C, Luscher TF, et al. Electrophysiological findings in patients with isolated left ventricular non-compaction. Europace: European pacing, arrhythmias, and cardiac electrophysiology: journal of the working groups on cardiac pacing, arrhythmias, and cardiac cellular electrophysiology of the European Society of Cardiology. 2009;11(9):1193-200.

22. Steffel J, Duru F. Rhythm disorders in isolated left ventricular noncompaction. Annals of medicine. 2012;44(2):101-8.

23. Fazio G, Corrado G, Zachara E, Rapezzi C, Sulafa AK, Sutera $L$, et al. Ventricular tachycardia in non-compaction of left ventricle: is this a frequent complication? Pacing and clinical electrophysiology. PACE. 2007;30(4):544-6.

24. Caliskan K, Szili-Torok T, Theuns DA, Kardos A, Geleijnse $M L$, Balk $A H$, et al. Indications and outcome of implantable cardioverter-defibrillators for primary and secondary prophylaxis in patients with noncompaction cardiomyopathy. Journal of cardiovascular electrophysiology. 2011;22(8): 898-904.

25. Ashrith G, Gupta D, Hanmer J, Weiss RM. Cardiovascular magnetic resonance characterization of left ventricular noncompaction provides independent prognostic information in patients with incident heart failure or suspected cardiomyopathy. Journal of cardiovascular magnetic resonance: official journal of the Society for Cardiovascular Magnetic Resonance. 2014; $16: 64$

26. van Dalen BM, Caliskan K, Soliman OI, Nemes A, Vletter WB, Ten Cate FJ, et al. Left ventricular solid body rotation in 
non-compaction cardiomyopathy: a potential new objective and quantitative functional diagnostic criterion? European journal of heart failure. 2008;10(11):1088-93.

27. Fernandez-Golfin C, Pachon M, Corros C, Bustos A, Cabeza B, Ferreiros $\mathrm{J}$, et al. Left ventricular trabeculae: quantification in different cardiac diseases and impact on left ventricular morphological and functional parameters assessed with cardiac magnetic resonance. Journal of cardiovascular medicine. 2009;10(11):827-33.

28. Dodd JD, Holmvang G, Hoffmann U, Ferencik M, Abbara S, Brady TJ, et al. Quantification of left ventricular noncompaction and trabecular delayed hyperenhancement with cardiac MRI: correlation with clinical severity. AJR American journal of roentgenology. 2007;189(4):974-80.

29. Nucifora G, Aquaro GD, Pingitore A, Masci PG, Lombardi M. Myocardial fibrosis in isolated left ventricular non-compaction and its relation to disease severity. European journal of heart failure. 2011;13(2):170-6.

30. Grothoff M, Pachowsky M, Hoffmann J, Posch M, Klaassen $S$, Lehmkuhl $L$, et al. Value of cardiovascular MR in diagnosing left ventricular non-compaction cardiomyopathy and in discriminating between other cardiomyopathies. European radiology. 2012;22(12):2699-709.

31. Kohli SK, Pantazis AA, Shah JS, Adeyemi B, Jackson G, McKenna WJ, et al. Diagnosis of left-ventricular non-compaction in patients with left-ventricular systolic dysfunction: time for a reappraisal of diagnostic criteria? European heart journal. 2008;29(1):89-95.

32. Di Lenarda A, Scherillo M, Maggioni AP, Acquarone N, Ambrosio GB, Annicchiarico $M$, et al. Current presentation and management of heart failure in cardiology and internal medicine hospital units: a tale of two worlds-the TEMISTOCLE study. American heart journal. 2003;146(4):E12.

33. Kobza R, Steffel J, Erne P, Schoenenberger AW, Hurlimann D, Luscher TF, et al. Implantable cardioverter-defibrillator and cardiac resynchronization therapy in patients with left ventricular noncompaction. Heart rhythm: the official journal of the Heart Rhythm Society. 2010;7(11):1545-9.

34. Murphy RT, Thaman R, Blanes JG, Ward D, Sevdalis E, Papra $E$, et al. Natural history and familial characteristics of isolated left ventricular non-compaction. European heart journal. 2005;26(2):187-92. 